\title{
Beneficial Effects of Regular Physical Exercises on Mental State in Visually Impaired and Deaf-blind Adults
}

\author{
Airi Surakka ${ }^{1, *}$, Mika Venojarvi ${ }^{1}$, Kauko Pitkanen ${ }^{2}$ \\ ${ }^{1}$ School of Medicine, Biomedicine/ Exercise Medicine, University of Eastern Finland, Finland \\ ${ }^{2}$ The Finnish Brain Research and Rehabilitation Center Neuron, Finland
}

Copyright $(\subset 2016$ by authors, all rights reserved. Authors agree that this article remains permanently open access under the terms of the Creative Commons Attribution License 4.0 International License

\begin{abstract}
The most common mental health problems in visually impaired and deaf-blind adults are depression, anxiety, tension, difficulties in falling asleep and disturbed sleep. The physical training program used in this study was designed with the aim of reducing the physical and mental problems of this population. Twenty-four visually impaired and deaf-blind adults completed a 5-6-week supervised physical training program (three 60-minute sessions per week) with varying rhythms of music. Motivation was enhanced in a number of ways, including measurement of weight, blood pressure, flexibility of upper body and trunk and gait analysis before and after the intervention. After completion of the training program the participants themselves assessed the effect of the intervention by answering freely in their own words the question: "Did you feel or notice any effect of the physical training program?" The responses showed that 22 participants experienced improvements in physical condition (binomial one-sided confidence interval $\mathrm{CI}=0.76$, confidence level 95\%), 21 in mental state $(\mathrm{CI}=0.71)$ and 11 in balance $(\mathrm{CI}=0.28)$. According to this study moderate and regular physical exercises reduce mental and physical problems and allow visually impaired and deaf-blind adults to live to the fullest with independent mobility, orientation, and better self-confidence and self-esteem.
\end{abstract}

Keywords Physical Exercises, Visually Impaired, Deaf-blind, Mental State

\section{Introduction}

People who are sedentary have more anxiety and depression, lower self-esteem, lower confidence levels, and poorer self-efficacy than people with a more active lifestyle [1]. Physical activity, body composition and perceived quality of life have been found to be lower in visually impaired adults than in normally sighted adults [2].

The most common mental problems of sedentary visually impaired and deaf-blind adults are depression, anxiety, difficulty in falling sleep, broken sleep and short sleep [3]. In a research study performed by the International Centre for Eye Health to investigate the connection between visual impairment, depression and anxiety, 13,900 persons over 75 years old were chosen randomly for participation. Their vision was examined, depression was assessed with the Geriatric Depression Scale (GDS-15) and anxiety was assessed with the General Health Questionnaire (GHQ-28). The results showed that problems of visually impaired persons in daily life often lead to depression [4]. In another study investigating the occurrence of depression in visually impaired people 75 years and older $(n=391)$ depression was found to be common in the group with severe visual impairment [5]. Donoyama and Takeda investigated mental health and related factors in 155 visually impaired massage practitioners and found that young masseurs had higher anxiety and depression levels than older masseurs [6]. A study amongst elderly Italian subjects showed that vision and hearing impairments were associated with depressive-anxiety syndrome [7].

Many individuals engaging in daily exercise report better ability to sleep, improved self-esteem, increased stamina and decreased stress levels [3]. Regular all-round physical exercises can improve the proprioceptive sensory system and enhance self-esteem, self-confidence, willpower, power of concentration and contribute to a 'relaxed mood' [8]. In a study conducted by Kabudzki et al. the international Physical Activity Questionnaire and Life Satisfaction Questionnaire were administered to a sample of eighty-two individuals (mean age 38 years) with varying degrees of vision loss, more than $50 \%$ of whom were highly physically active. The results showed that the level of physical activity was significantly positively correlated with the level of life satisfaction and that physical activity increased the subjective quality of life in blind and visually impaired individuals [9].

According to Anderson and Shivakumar people who engage in regular physical activity have fewer depressive and anxiety symptoms, because physiological changes 
enhance synthesis and liberation of neurotrophic factors, cognitive functioning, angiogenesis, neuroplasticity, synaptogenesis, neuroprotection, and endorphin and monoamine production and decrease oxidative stress and cortisol excretion [10]. These positive physiological changes improve mental state, making independent orientation and mobility easier, and thus also improve quality of daily life in visually impaired and deaf-blind adults.

In the present study visually impaired and deaf-blind adults were motivated to perform regular physical exercises in many ways: performance of the physical exercises in small groups, ideal lighting and a low echo level in the gymnasium (important for subjects with hearing aids), special instruction methods, a relaxed atmosphere during training sessions, a specially designed physical training program which would also be easy to perform at home after the intervention, the possibility of always choosing different training music at home, take home instructions in a form suited to each subject to enable continuation of the physical training program after the intervention [11]. To the best of our knowledge, there are no physical exercise programs specially designed for common mental and physical problems of visually impaired and deaf-blind adults. Furthermore there are no intensive physical training interventions including assessments of the effects and no studies of physical interventions for visually impaired and deaf-blind people with special arrangements: transport service to training sessions and home if necessary, lighting, echo level induction loop with two microphones (one in the chest of the instructor and another in front of record player). In addition the instructor was well educated for rehabilitation of visually impaired and deaf-blind people. The instructor knew sight and hearing level of each participant which helped to instruct and communicate with participants. It was important that a group of participants in training sessions was small (6-7 participants) so the instructor was able to see each participant. The training group consisted of visually impaired and deaf-blind participants. The purpose of the study was to arrange the setting and instruction as easy as possible to each participant so that they are able to concentrate on physical exercises only and to investigate the effects of intervention on mental state in visually impaired and deaf-blind adults. Another purpose was to motivate participants to perform regular physical exercises after the intervention.

\section{Methods}

\subsection{Participants}

Twenty-four participants (31-75 years, mean age 53 years, 12 with and 12 without previous physical activity) completed the physical training program. All participants were volunteers from Helsinki University Rehabilitation Clinic. Fifteen participants (13 females and 2 males) were classificated as partially sighted, six participants were classified as blind (3 females and 3 males). Diagnoses of partially sighted and blind participants were degeneration maculae and dystrophia retinae pigmentosa. Three participants were classified as deaf-blind. Their diagnose was Usher syndrome (dystrophia retinae pigmentosa and sensory neural hearing loss). Sign language was not needed during training sessions in spite of their profound hearing loss because of their strong hearing aids with telephone setting and induction loop with two microphones. A prerequisite for participation in the study was the absence of disabilities or diseases that might prevent effective physical training and completion of the study. In the interview of participants the most important motive for participating in the intervention was "better physical condition", followed in second place by the possibility to participate into an intervention organized specially for visually impaired and deaf-blind people with peers. We think that our small sample of participants results information enough of the effects of physical exercises as a pilot study.

\subsection{Procedure}

The physical exercises were performed at moderate intensity (5-6 weeks, three 60-minute sessions per week) and were designed to reduce the most common physical problems in this population: tension in neck and shoulder muscles, balance, coordination and flexibility of upper body and trunk [11]. An important pre-requisite of the physical training program was that it should be easy to perform at home after completion of the intervention. Transportation from home to the gymnasium and back was organized for all subjects needing it. In this way participants were able to concentrate only on performing the physical exercises. Physical training program was performed with changing rhythm of music. It consisted warm-up exercises, eight special exercises with repetitions, stretching exercises and relaxation

\subsection{Ways of Motivating}

Motivation for physical exercise was enhanced in several ways. Before the intervention the instructor met each participant individually. She performed measurements such as weight, height and blood pressure and informed the participants at the same time about the benefits of regular physical exercise. The participants had the opportunity to ask questions. After the intervention all participants spent a further two days together during which they performed the exercise program of the study again. The instructor also had a meeting with each subject individually at which she told them and explained their individual results. Each participant was given a printout of the results to take home. At the meeting of all participants the instructor explained the results of the group as a whole and informed them again about the benefits of regular physical exercise as show by the results of other studies.

The physical training program was performed in small 
groups (6-7 subjects) with changing rhythms of music. In the gymnasium the echo level was designed to be low (important for subjects with hearing aids). The artificial lighting could be diminished or increased in different parts of the gymnasium depending on the visual requirements of the participants. Special instruction methods were used, for example by using descriptive words or describing the direction of movement on the basis of the clock face. Exercises were described verbally and simultaneously demonstrated in good lighting, with the instructor wearing dark clothes and shoes and standing in front of a light wall. Each exercise had its own name which helped to memorize the exercise [11]. All participants exercised inside an induction loop. This allowed participants with hearing aids to hear verbal instructions and music without interference from surrounding noise. Motivation was also enhanced through several measurements before and after the intervention, including weight, blood pressure, flexibility of trunk and upper body and gait analysis.

\section{Results}

We chose a simpler way to assess the mental effect of the physical training program than with the methods commonly in use, for example The Satisfaction With Life Scale (SWLS) [12]. SWLS contains five statements that you may agree or disagree by using the 1-7 scales indicating the agreement with each item. Our group of participants, visually impaired and deaf-blind people, is a special group. We normally sighted people want to see and take your time to consider all options of SWLS at the same time for being able to find the most suitable alternative. Visually impaired and deaf-blind people often need another person to read all multiple choices out loud and in addition some of the participants have difficulties in hearing. Furthermore the life satisfaction statements related to pre- and post-test items are more difficult to assess than assessment of the change in these items related to the intervention.

Therefore after intervention the participants assessed the effect of the intervention by responding freely with their own words to one prompt question: "Did you feel or notice any effect of the physical training program?" The question was presented without any explanation in order to receive spontaneous responses. In this way we found out any unexpected, individual and the most important effects to each subject. Their answers were classified into three groups: physical condition, mental state and balance. The responses showed that 22 of 24 subjects with and without previous physical activities experienced improvements in physical condition (binomial one-sided confidence interval $\mathrm{CI}=0.76$ with confidence level 95\%), 21 subjects in mental state (CI = 0.71 ) and 11 subjects in balance (CI 0.28).

Individual effects of the intervention were expressed in various ways. For example, two participants reported that dizziness disappeared (= balance improved), while others actually used the expression: balance improved. Improved physical condition was expressed in different ways, for example, 'my hip is more flexible', 'my neck and shoulder muscles are more relaxed', it is easier to walk upstairs', 'my back is better', 'I sleep better'. These physical effects also had a positive influence on mental state which was also expressed in different ways, for example, 'the exercises had a bracing effect', 'my mind feels lighter', 'I feel lighter, I feel better'. Self-assurance increased, for example after some training sessions one participant asked to be shown where the bus stop was. She wanted to use public transport. She had always come to the training sessions by taxi but now she felt that she was able to use public transport.

All participants were asked by telephone about physical activity three months after the termination of the intervention. Everybody was still performing regular physical activities.

\section{Discussion and Conclusion}

The current study was designed to motivate visually impaired and deaf-blind participants to perform physical exercises regularly because of their mental, physical and physiological benefits. The participants expected better physical condition as a result of the intervention but it was surprising to find that 21 participants also experienced improvements in mental state. One purpose of the study was to show that physical exercises can be demanding and at the same time enjoyable and fun with varying music. Each participants started exercises from her/his own physical level without stress. The feeling of mastering a demanding exercise program as well as normally sighted persons in spite of visual or hearing-visual impairment improved self-esteem. The physical training program was easy to perform at home in a small room with self-selected music after the intervention. An induction loop is also available at home for hearing aid users. Three months after the intervention all participants were still performing various kinds of regular physical exercises.

Stanton and Reaburn conducted a systematic review of exercise program variables in the treatment of depression. They concluded that supervised aerobic exercises undertaken three times weekly at moderate intensity for a minimum of nine weeks were the most recommendable kind of exercise program in the treatment of depression [14]. Our physical exercise intervention consisted of three supervised 60-minute sessions per week for 5-6 weeks. We thought that this intervention was long enough for participants to feel positive changes in their body and mental state which would motivate them to continue the physical exercises after the intervention. The results showed positive psychological and physical changes which motivated participants to continue physical exercises after the intervention at home and outside the home. According to this study moderate and regular physical exercises reduced the most common mental problems of visually impaired and deaf-blind adults in spite 
of age, gender, level of sight or hearing, previous physical activity or inactivity. Each participant had her/his individual situation of sight and hearing but 21 felt that her/his mental well-being improved.

There are several possible reasons for the improved mental state of participants. Regular physical exercise enhances self-confidence, power of concentration, self-esteem and self-efficacy. Positive changes in the participant' physical condition, such as dynamic balance in walking, reduced tension in neck and shoulder muscles and flexibility of upper body and trunk, also had a positive influence on mental state. During the training sessions the participants' minds were diverted from negative thoughts because they had to concentrate on the exercises. They noticed that they successfully mastered the demanding exercise program in spite of visual impairment and deaf-blindness or age. This was possible when training sessions were performed in small groups with ideal lighting, low echo level, the use of induction loop and good contrasts. The social contacts made the participants more cheerful. It was interesting, that they encouraged spontaneously each other to perform exercises better. The atmosphere during the exercise sessions was relaxed and interactive. The 'Mastery Climate and Target Structure' described by Epstein was similar to that used in these physical sessions in terms of tasks, authority, recognition, grouping, evaluation and time [13].

Limitation of the study was a small sample size but in the future it would be interesting to organize an intervention of 2-3 months with bigger sample size with the same frequency of training sessions per week with interim tests. Questioning about regular physical activities after the intervention should also be done later, for example after half a year and after a year. This would enable us to find out if regular physical exercises have become part of the participants' way of life, which was the main purpose of our intervention.

\section{REFERENCES}

[1] Morgan, W. (1994). Physical activity, fitness and depression. In Bouchard, C., Shephard, R., \& Stephens, T. [eds.] Physical activity, fitness and health: International proceedings and consensus statement. Champaign, IL: Human Kinetics. 851-867.

[2] Holbrook, E., Caputo, J., Perry, T., Fuller, D., Morgan, D. (2009). Physical activity, body composition and perceived Quality of life of adults with visual impairments. J Visual Impair Blin., 10, 17-29.

[3] Martinsen, E., \& Stephens, T. (1994). Exercise and mental health in clinical and free-living populations. In Dishman R. (ed.). Advances in exercise adherence. Champaign, IL: Human Kinetics. 55-72.

[4] Evans, J., Fletcher, A., Wormald, R. (2007). Depression and anxiety in visually impaired older people. Ophthalmology, $114,283-288$.

[5] Hayman, K., Kerse, N., La Grow, S., Robertson, M., Cambell, A. (2007). Depression in older people: visual impairment and subjective health. Optom Vis Sci., 84, 1024-1030.

[6] Donoyama, N., Takeda, F. (2007). Mental health and related factors among massage practitioners with visual impairment. Industrial Health, 45, 191-198.

[7] Bernebei, V., Moretti, F., Marchiori, A., Ferrari, B., Dalmonte, E., De Ronchi, D., \& Atti, A. (2011). Vision and hearing impairments are associated with depressive-anxiety syndrome in italian elderly. Aging Ment Health, 15, 467-474.

[8] Lieberman, L., \& Houston-Wilson (1999). 'Overcoming the Barriers to Including Students with Visual Impairments and Deaf-blindness in Physical Education', Review, 31, 129-138.

[9] Kabudzki, Jakub, Tasiemski, Tomasz (2013). Physical Activity and Life Satisfaction in Blind and Visually Impaired Individuals. Human Movement, 14, 210-216.

[10] Anderson, E., \& Shivakumar, G. Effects of Exercise and Physical Activity on Anxiety. doi: 10 3389/fpsyt.2013.00027

[11] Surakka, A., Kivelä, T. (2008). Motivating visually impaired and deaf-blind people to perform regular physical exercises. British Journal of Visual Impairment, 26, 255-268.

[12] Pavot, W., Diener, E. (1993). Review of the Satisfaction with Life Scale. Psychological Assessment, 5, 164-172.

[13] Biddle, S. (2001). 'Enhancing Motivation in Physical Education', in C. G. Roberts (ed.) Advances in Motivation in Sport and Exercise, 121-127. USA: Human Kinetics Publishers.

[14] Stanton, R., \& Reaburn, P. Exercise and the treatment of depression: A review of the exercise program variables. doi: 10.1016/j.jsams.2013.03.010 